Gut and Liver, Vol. 10, No. 1, January 2016, pp. 109-116

\title{
Clinical Implications of the Serum Apelin Level on Portal Hypertension and Prognosis of Liver Cirrhosis
}

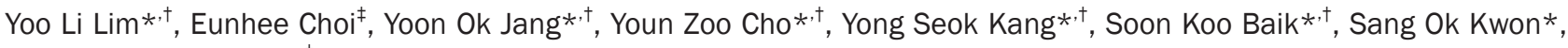 \\ and Moon Young Kim*, ${ }^{*}$ \\ Departments of *Internal Medicine, ${ }^{\dagger}$ Cell Therapy and Tissue Engineering; Wonju Severance Christian Hospital, Yonsei University Wonju \\ College of Medicine, and ${ }^{\ddagger}$ Institute of Lifestyle Medicine, Yonsei University Wonju College of Medicine, Wonju, Korea
}

Background/Aims: Levels of serum apelin (s-apelin), an endogenous ligand for angiotensin-like receptor 1 , have been shown to be related to hepatic fibrosis and hemodynamic abnormalities in preclinical studies. We investigated the clinical implications of s-apelin as a noninvasive prognostic biomarker for chronic liver disease (CLD). Methods: From January 2009 to December 2012, 215 CLD patients were enrolled and underwent clinical data collection, hepatic venous pressure gradient (HVPG) measurement, and liver biopsy. sapelin was detected with a human total apelin enzyme-linked immunosorbent assay kit. All patients were prospectively observed during the median follow-up period of $23.0 \pm 12.9$ months for decompensation and mortality. Results: A total of 42 patients (19.5\%) died during the follow-up period. s-apelin was significantly correlated with measurements of liver stiffness $\left(R^{2}=0.263, p<0.001\right)$ and collagen proportional area $\left(R^{2}=0.213, p<0.001\right)$ measured from liver biopsy tissue and HVPG $\left(R^{2}=0.356, p<0.001\right)$. In a multivariate analysis using a Cox regression hazard model, s-apelin was a weakly significant predictor of decompensation (hazard ratio [HR], 1.002; $p<0.001)$ and mortality (HR, 1.003; $p<0.001)$. Conclusions: s-apelin showed a significant relationship with CLD severity. However, its significance as a noninvasive biomarker for disease severity and prognosis was weak. (Gut Liver 2016;10:109-116)

Key Words: Apelin; Portal hypertension; Liver cirrhosis; Prognosis; Biological markers

\section{INTRODUCTION}

Chronic liver disease (CLD) including cirrhosis is caused by various etiologies, such as hepatitis viruses and alcohol. Through these stimulation and inflammatory injury, intrahepatic fibrotic change begins and is followed by intrahepatic vascular change and angiogenesis and portal hypertension (PHT) development. As fibrotic change keep going, intrahepatic changes progress to systemic hemodynamic abnormality, hyperdynamic circulatory syndrome. Finally, liver cirrhosis includes heterogeneous clinical manifestations and stages related with various histological and hemodynamic changes. ${ }^{1,2}$

In compensated CLD, the clinical focus is to terminate the chronic intrahepatic inflammation or inflammatory response. However, once the disease progress to decompensated state, not only intrahepatic histological state but also extrahepatic and systemic hemodynamic circulatory problems related with PHT also become an important factor which should be considered. ${ }^{3-5}$ Therefore, the estimation of both intrahepatic and systemic hemodynamic aspects of CLD is strongly recommended in the management of patients. As known well, the gold-standard method for the diagnosis of liver histology and hemodynamics are liver biopsy and hepatic venous pressure gradient (HVPG) measurement. However, both biopsy and HVPG have limitations in routine clinical application including invasiveness and difficulty in repeated measurement. ${ }^{6-8}$ So the strong need for new noninvasive biomarker that is helpful in both histological and hemodynamic estimation has been for a long time. In this point serum apelin (s-apelin) has been suggested as a good candidate.

In 1993, O'Dowd et al. ${ }^{9}$ discovered APJ, an orphan G proteincoupled receptor with structural similarity to the angiotensin

\footnotetext{
Correspondence to: Moon Young Kim

Department of Internal Medicine, Wonju Severance Christian Hospital, Yonsei University Wonju College of Medicine, 20 Ilsan-ro, Wonju 26426, Korea

Tel: +82-33-7411229, Fax: +82-33-7451228, E-mail: drkimmy@yonsei.ac.kr

Yoo Li Lim and Eunhee Choi equally contributed to this study.

Received on September 5, 2014. Revised on November 15, 2014. Accepted on November 18, 2014. Published online May 13, 2015

pISSN 1976-2283 eISSN 2005-1212 http://dx.doi.org/10.5009/gnl14345

(a) This is an Open Access article distributed under the terms of the Creative Commons Attribution Non-Commercial License (http://creativecommons.org/licenses/by-nc/4.0) which permits unrestricted non-commercial use, distribution, and reproduction in any medium, provided the original work is properly cited.
} 
receptor, and endogenous ligand for APJ, apelin was found in 1998. ${ }^{10}$ Apelin is distributed across numerous organs, including the brain, liver, heart, spleen, kidney, and lung. Through many previous studies, apelin has been known to induce decreases of systemic arterial and venous pressure by increase of nitric oxide (NO) production. Apelin also involves in cardiovascular system, water homeostasis and electrolyte control. ${ }^{11-13}$ In several preclinical studies with cirrhotic animal model, s-apelin showed close relationships with both intrahepatic fibrosis and splanchnic hemodynamics. ${ }^{14-16}$ However, clinical data that shows the associations between s-apelin and histological and hemodynamic severity is very limited. Therefore, in this study, we aimed to investigate the clinical relationship of s-apelin in CLD patients and its possibility as prognostic biomarker in the both aspect of hepatic fibrosis and systemic hemodynamic change.

\section{MATERIALS AND METHODS}

\section{Patients}

Subjects were drawn from a consecutive series of 353 cirrhotic patients who were admitted to Wonju Severance Christian Hospital, Yonsei University Wonju College of Medicine, between January 2009 and December 2012. The diagnosis of CLD and cirrhosis was confirmed by liver biopsy in 172 patients and by the presence of varices in the esophagogastroduodenoscopy, laboratory data, or image studies, including ultrasonography and computer tomography scans, in the others. Patients who did not provide informed consent or had hepatocellular carcinoma, other malignancies within the past 3 years, severe hepatic failure (serum bilirubin level $>5 \mathrm{mg} / \mathrm{dL}$ or hepatic encephalopathy), thrombosis in the inferior vena cava or hepatic or portal vein, uncontrolled infectious conditions (such as spontaneous bacterial peritonitis or sepsis), heart failure greater than the New York Heart Association class III, acute renal failure, or severe chronic renal failure (eGFR $<30 \mathrm{~mL} / \mathrm{min} / 1.73 \mathrm{~m}^{2}$ ), uncontrolled hypertension, pregnancy or lactation, or any other medical or psychiatric problems deemed to be unsuitable for clinical study were excluded. After all exclusions, 215 patients were ultimately enrolled in this study (Fig. 1). For 215 patients, the measurement of HVPG, liver stiffness measurement (LSM) using transient elastography (Fibroscan ${ }^{\circledR}$; Echosens, Paris, France), and baseline s-apelin were undertaken with general basic serologic and radiologic tests. For the patients who were undertaken liver biopsy, the measurement of collagen proportion area (CPA) also was performed. All enrolled patients were prospectively observed until December 31, 2013. During the follow-up period, the patients visited the hospital every 3 months and were observed for the development of variceal bleeding, hepatic failure, hepatic encephalopathy, other liver related events, and mortality. This study was approved and supervised by the Institutional Review Board of the Yonsei University Wonju College of Medicine.

\section{2. s-apelin measurement}

Blood samples were obtained from the peripheral vein 1 hour before HVPG measurement. Immediately after sampling, the serum was separated from the blood and stored at $-80^{\circ} \mathrm{C}$ until the analysis. The human total apelin ELISA kit (MyBioSource, San Diego, CA, USA) was used to measure s-apelin, according to the manufacturer's suggestions. The 96-well microplate was coated with the apelin-specific monoclonal antibody. The eight different concentrations of the standard material, positive and negative control material and $50 \mu \mathrm{L}$ of each patient's serum were transferred into each well of the ELISA plate. A total of $100 \mu \mathrm{L}$ of conjugate solution attached to horse radish peroxidase was added to each well, and the samples were left to react for 1 hour at $37^{\circ} \mathrm{C}$ after mixing thoroughly. After the reaction, the samples were washed with washing buffer 5 times to remove unbound substances. Then, $100 \mu \mathrm{L}$ of the substrate solution was added to each well, and the plate was kept in the dark for 10 to 15 minutes at $37^{\circ} \mathrm{C}$. The reaction was suspended with $50 \mu \mathrm{L}$ of stop solution, and the absorbance at $450 \mathrm{~nm}$ light wave was measured in each well with a microplate reader (BioTek ELX 800; BioTek, Shoreline, WA, USA). A standard curve was calculated using the blank and eight different concentrations of the standard material, and this curve was used to convert the absorbance of each sample to the s-apelin.

\section{HVPG measurement}

After an overnight fast, the right hepatic vein (HV) was catheterized percutaneously through the femoral vein, and the pressure was recorded in both the free and the wedged position with a 7F balloon-tipped catheter (Arrow International, Erding, Germany). HVPG was determined by subtracting the free HV

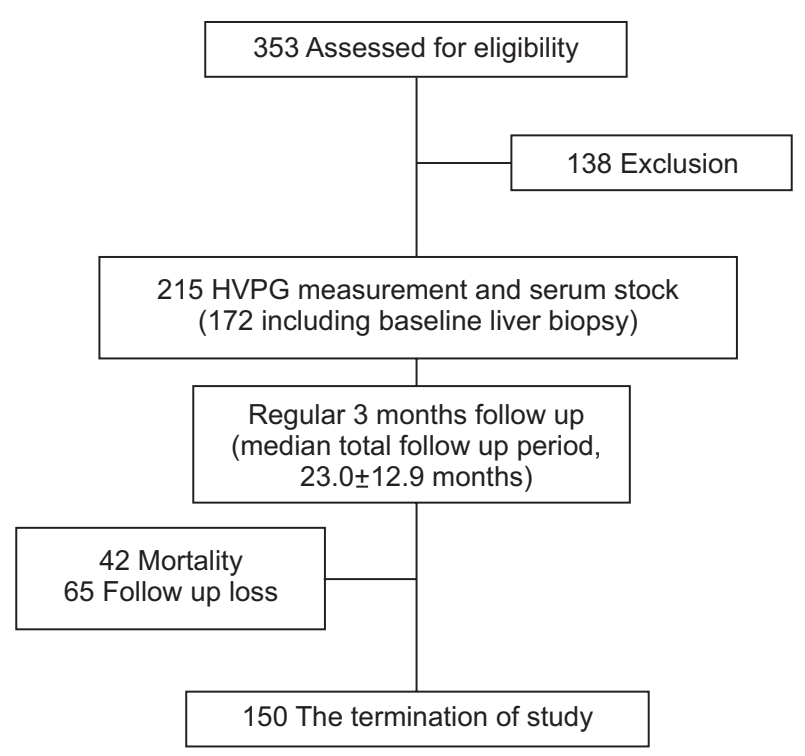

Fig. 1. Study flow chart. HVPG, hepatic venous pressure gradient. 
pressure from the wedged HV pressure. ${ }^{7,17}$ All measurements were performed in at least triplicate, and permanent tracings were obtained on a multichannel recorder. An examiner (Y.J.K.) with more than 10 years of experience in HVPG measurement performed all of the HVPG procedures without any clinical information. The coefficient of variation in HVPG measurement at our medical center was 7\%. Clinically significant portal hypertension (CSPH) was defined as an HVPG value $\geq 10 \mathrm{~mm} \mathrm{Hg}$, according to a consensus definition. ${ }^{18}$

\section{Liver stiffness measurement}

LSM was performed with a transient elastography (Fibroscan ${ }^{\circledR}$; Echosens). This device consists of a 3.5-MHz ultrasound transducer $\mathrm{M}$ probe mounted on the axis of a vibrator. Mild amplitude and low-frequency vibrations $(50 \mathrm{~Hz})$ are transmitted to the liver tissue, inducing an elastic shear wave that propagates through the underlying liver tissue. LSM was performed on right lobe of the liver; in the intercostal space with the patient lying in dorsal decubitus with the right arm at maximal abduction. The operator assisted by an ultrasonic time-motion image, located a liver portion at least $6 \mathrm{~cm}$ thick and free of large vascular structures and the gallbladder. ${ }^{19}$ The LSM was assessed on the same day as the HVPG measurement by a single examiner (Y.Z.C.) with 5 years of experience in LSM in patients with CLD without any clinical information. LSM values are expressed in kilopascal $(\mathrm{kPa})$, were calculated as the median value of ten successful acquisitions, and were considered reliable based on a success rate of at least $60 \%{ }^{20}$

\section{CPA measurement}

CPA was measured through the histological analysis of the liver biopsy specimen which was obtained within 6 months from study enrollment. Liver biopsies were performed percutaneously with an 18-gauge needle (TSK ACECUT biopsy needle; TSK Laboratory, Tochigi, Japan). Each specimen sized at least 15 $\mathrm{mm}$ in length, and at least $1.2 \mathrm{~mm}$ in width. Five-micrometer thick sections of paraffin-embedded liver biopsy specimens were deparaffinized and rehydrated with distilled water and stained with a Picrosirius red staining kit (Polysciences, Warrington, PA, USA) according to the manufacturer's suggestions. In addition, the amount of collagen (the main component of fibrous tissue) was estimated from the CPA, expressed as the percentage of the total area that was positive for Picrosirius red stain on microscopy (Olympus BX51; Olympus, Tokyo, Japan) using a computerized image-analysis system (IMT i Solution, Vancouver, Canada). While measuring the CPA, we eliminated image artifacts and structural collagen in large portal tracts and blood vessel walls. ${ }^{21}$ The CPA also was measured by a single examiner (Y.O.J.) without any clinical information.

\section{Statistical analysis}

Continuous variables are expressed as means \pm standard de- viation. One-way analysis of variance was used to analyze the relationships between s-apelin and the Child-Turcotte-Pugh's (CTP) class. Pearson correlation was used to analyze the relationships between s-apelin and the Model for End-stage Liver Disease (MELD) score, LSM, CPA, and HVPG. To evaluate the diagnostic value of s-apelin, area under receiver operating curve (AUROC) was also analyzed. Especially, the result of comparison

Table 1. General Patient Characteristics ( $n=215)$

\begin{tabular}{|c|c|}
\hline Characteristic & Value \\
\hline Age, yr & $50.8 \pm 8.8$ \\
\hline Sex, male:female & $185: 30(86: 14)$ \\
\hline \multicolumn{2}{|l|}{ Etiology } \\
\hline Alcohol & $155(72.1)$ \\
\hline HBV & $36(16.7)$ \\
\hline Alcohol and HBV & $12(5.6)$ \\
\hline $\mathrm{HCV}$ & $3(1.4)$ \\
\hline Alcohol and HCV & $2(0.9)$ \\
\hline Cryptogenic & $7(3.3)$ \\
\hline Platelet count, $10^{9} / \mathrm{L}$ & $128 \pm 86$ \\
\hline AST, U/L & $59.7 \pm 32.3$ \\
\hline ALT, U/L & $33.8 \pm 28.5$ \\
\hline Albumin, g/dL & $3.4 \pm 0.5$ \\
\hline Total bilirubin, $\mathrm{mg} / \mathrm{dL}$ & $1.7 \pm 2.5$ \\
\hline PT, \% & $80.8 \pm 18.6$ \\
\hline $\mathrm{Na}, \mathrm{mmol} / \mathrm{L}$ & $138.0 \pm 5.2$ \\
\hline Creatinine, $\mathrm{mg} / \mathrm{dL}$ & $0.78 \pm 0.49$ \\
\hline s-apelin, pg/mL & $863.6 \pm 219.7$ \\
\hline \multicolumn{2}{|l|}{ Child-Turcotte-Pugh's class } \\
\hline $\mathrm{A}$ & $117(54.4)$ \\
\hline B & $84(39.1)$ \\
\hline $\mathrm{C}$ & $14(6.5)$ \\
\hline MELD score & $10.1 \pm 3.7$ \\
\hline Compensation:decompensation & 103:112 (47.9:52.1) \\
\hline \multicolumn{2}{|c|}{$\begin{array}{l}\text { Liver histology in biopsy (METAVIR score } \\
{[\mathrm{n}=172] \text { ) }}\end{array}$} \\
\hline $\mathrm{F} 1$ & $7(4.1)$ \\
\hline $\mathrm{F} 2$ & $11(6.4)$ \\
\hline F3 & $16(9.3)$ \\
\hline $\mathrm{F} 4$ & $138(80.2)$ \\
\hline LSM, kPa & $39.3 \pm 1.5$ \\
\hline СРА, \% & $26.4 \pm 1.5$ \\
\hline HVPG, mm Hg & $14.3 \pm 0.4$ \\
\hline
\end{tabular}

Data are presented as mean \pm SD or number (\%).

$\mathrm{HBV}$, hepatitis B virus; HCV, hepatitis C virus; AST, aspartate aminotransferase; ALT, alanine aminotransferase; PT, prothrombin time; sapelin, serum apelin; MELD score, model for end-stage liver disease score; LSM, liver stiffness measurement; CPA, collagen proportion area; HVPG, hepatic venous pressure gradient. 
of area under the curves performed using SAS version 9.2 (SAS Inc., Cary, NC, USA) and the comparison of time dependent AUROCs performed using $\mathrm{R}$ package. A multivariate analysis of the factors predicting decompensation and mortality was achieved using a Cox regression hazard model, and the predictive value for survival according to s-apelin was analyzed using KaplanMeier analysis. p-values of less than 0.05 indicated statistically significant differences. Statistical analysis was performed with IBM SPSS version 20.0 (IBM Co., Armonk, NY, USA).

\section{RESULTS}

\section{General characteristics}

The mean age was $50.8 \pm 8.8$ years, and 185 patients (86\%) were male and the most frequent cause of CLD was alcohol (155, $72.1 \%$ ). The median follow-up period was $23.0 \pm 12.9$ months, and 42 patients (19.5\%) expired during the follow-up period due to liver-related events. Among 103 patients who were initially diagnosed as compensated CLD, 17 patients (16.5\%) showed development of decompensation. The characteristics of these patients are summarized in Table 1.

\section{2. s-apelin and the clinical indices for the severity of liver cirrhosis}

The mean s-apelin according to the CTP class of A to C were 807.1 $\pm 239.9 \mathrm{pg} / \mathrm{mL}, 913.7 \pm 184.7 \mathrm{pg} / \mathrm{mL}$, and 933.8 $\pm 169.7 \mathrm{pg} /$ $\mathrm{mL}$, respectively. The s-apelin in the CTP class A were significantly lower compared with those of class B and C $(p<0.001)$; however, there was no significant difference between class $B$ and $C(p=0.947)$. s-apelin showed a significant linear correlation with CTP score (Pearson correlation coefficient $\left(\mathrm{R}^{2}=0.079\right.$, $\mathrm{p}<0.001)$. s-apelin also showed a significant linear correlation with MELD score $\left(\mathrm{R}^{2}=0.1, \mathrm{p}<0.001\right)$.

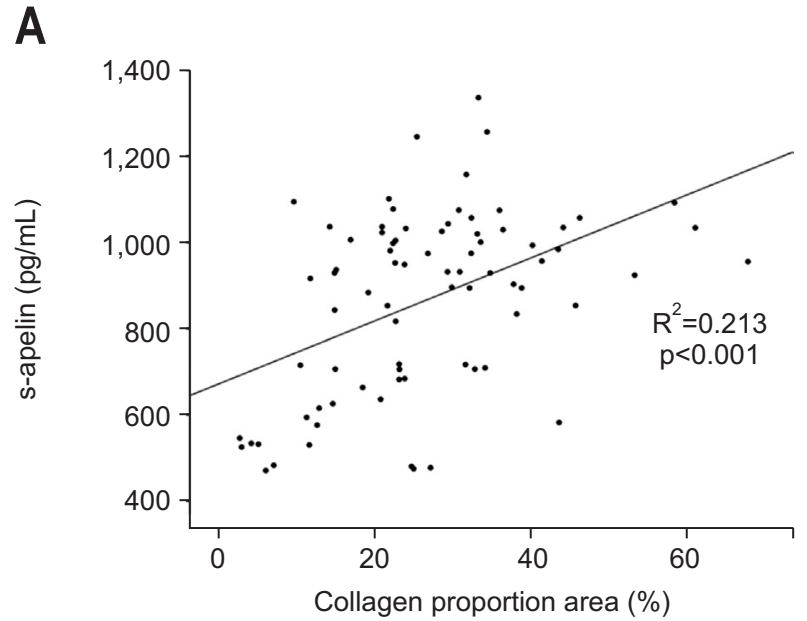

\section{3. s-apelin and the histological and hemodynamic state}

The histological state of liver cirrhosis was measured by two methods. The first was LSM by transient elastography, and the second was a direct CPA measurement. The mean values of LSM and CPA were $39.3 \pm 1.5 \mathrm{kPa}$ and $26.4 \% \pm 1.5 \%$, respectively. s-apelin showed a significant linear correlation with LSM $\left(\mathrm{R}^{2}=0.263, \mathrm{p}<0.001\right)$. s-apelin also showed a significant increase depending upon the increased fibrotic component of the liver, as presented by the CPA $\left(\mathrm{R}^{2}=0.213, \mathrm{p}<0.001\right)$ (Figs $2 \mathrm{~A}$ and 3$)$. Among the AUROCs for the prediction of histological cirrhosis (METAVIR score F4) of CTP score (0.632), MELD score (0.696),

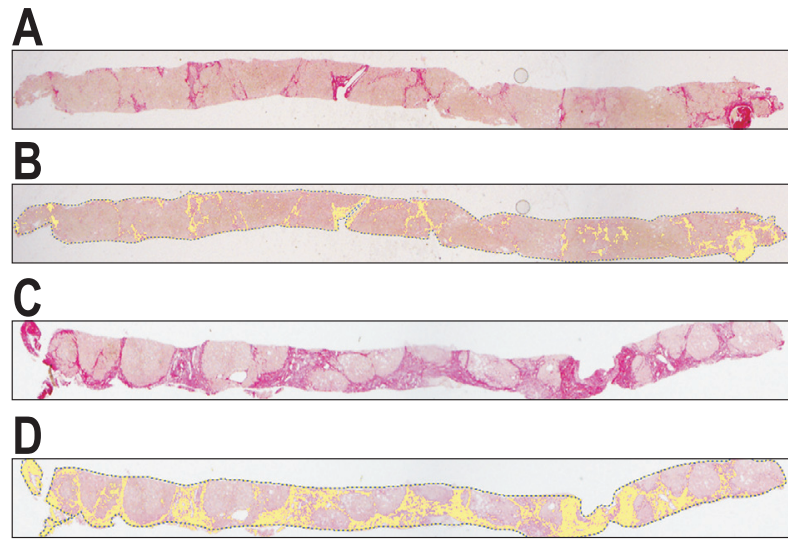

Fig. 3. Histologic findings of liver biopsy and collagen proportional area (CPA) measurements. (A) Picrosirius red staining $(\times 40)$ and $(B)$ computerized image analysis findings $(\times 40)$ in a patient with METAVIR F3 stage disease, CPA 6.90\%, hepatic venous pressure gradient (HVPG) $6 \mathrm{~mm} \mathrm{Hg}$, and serum apelin level (s-apelin) $483.07 \mathrm{pg} / \mathrm{mL}$. (C) Picrosirius red staining $(\times 40)$ and (D) computerized image analysis findings $(\times 40)$ in a patient with METAVIR F4 stage disease, CPA 29.30\%, HVPG $12 \mathrm{~mm} \mathrm{Hg}$, and s-apelin $1042.79 \mathrm{pg} / \mathrm{mL}$.

B

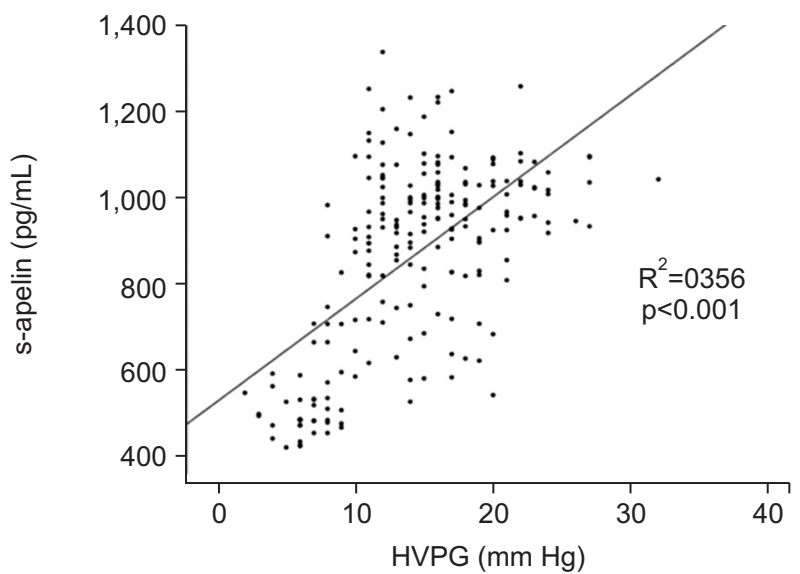

Fig. 2. Correlation of the serum apelin (s-apelin) level with collagen proportional area (CPA) and hepatic venous pressure gradient (HVPG). (A) A significant increase in s-apelin was dependent on an increase in the fibrotic component of the liver, as shown by the CPA (Pearson correlation coefficient $\left.\left[R^{2}\right]=0.213, p<0.001\right)$. (B) $s$-apelin also showed a significant linear correlation with HVPG $\left(R^{2}=0.356, p<0.001\right)$. 

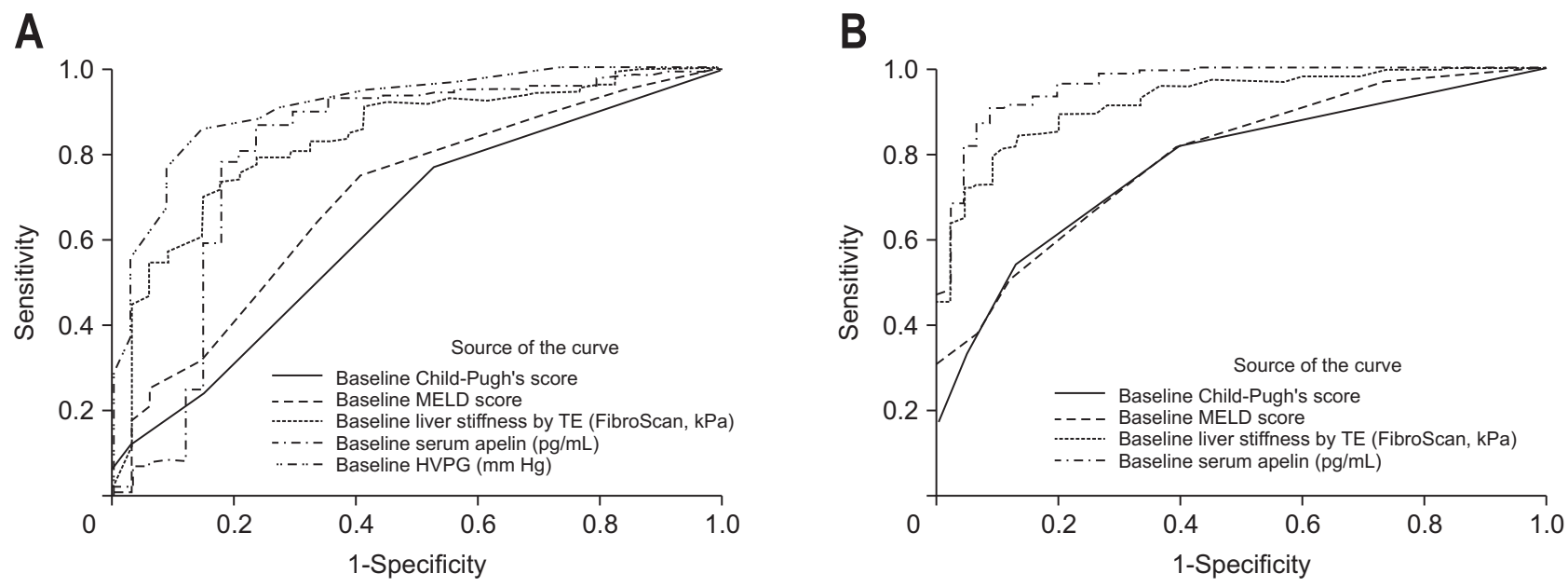

Fig. 4. Comparison of the area under receiver operating curves (AUROCs) for the prediction of histological cirrhosis (METAVIR stage F4) and clinically significant portal hypertension (CSPH). (A) The AUROCs for the prediction of histological cirrhosis (METAVIR score F4) of the Child-TurcottePugh's (CTP) score, the Model for End-stage Liver Disease (MELD) score, and liver stiffness measurement (LSM), the serum apelin (s-apelin) and the hepatic venous pressure gradient (HVPG) were $0.632,0.696,0.835,0.802$, and 0.919 , respectively. The AUROC of s-apelin showed significantly better diagnostic capacity than did the CTP score (CTP score vs s-apelin, $\mathrm{p}=0.006$ ). However, there was no difference in predictive power between the $s$-apelin and MELD scores ( $\mathrm{p}=0.0878$ ) or LSM ( $\mathrm{p}=0.4231$ ). The AUROC of HVPG was better than $\mathrm{s}$-apelin in the prediction of cirrhosis ( $\mathrm{p}=0.0061)$. (B) The AUROCs for the prediction of CSPH of the CTP score, MELD score, LSM, and s-apelin were 0.775, 0.793, 0.924, and 0.962, respectively. The AUROC of s-apelin showed a significantly better diagnostic ability than the CTP and MELD scores $(\mathrm{p}<0.001)$ and was similar to the AUROC of $\operatorname{LSM}(\mathrm{p}=0.108)$.

Table 2. Cox Regression Hazard Analysis for Parameters Related to Decompensation and Mortality

\begin{tabular}{lccc}
\hline \multicolumn{1}{c}{ Parameter } & HR & $95 \%$ CI & p-value \\
\hline Univariate analysis & & & \\
s-apelin, pg/mL & - & - & $<0.001$ \\
CTP score & - & - & $<0.001$ \\
MELD score & - & - & $<0.001$ \\
LSM, kPa & - & - & $<0.001$ \\
HVPG, mm Hg & - & - & $<0.001$ \\
Multivariate analysis*, & & & \\
Decompensation & & & \\
s-apelin, pg/mL & 1.002 & $1.000-1.006$ & 0.037 \\
CTP score & 0.960 & $0.624-1.478$ & 0.855 \\
LSM, kPa & 1.024 & $0.993-1.057$ & 0.668 \\
HVPG, mm Hg & 1.028 & $0.906-1.166$ & 0.093 \\
Mortality & & & \\
s-apelin, pg/mL & 1.003 & $1.001-1.005$ & 0.012 \\
CTP score & 1.223 & $0.971-1.564$ & 0.085 \\
LSM, kPa & 1.013 & $0.992-1.034$ & 0.237 \\
HVPG, mm Hg & 1.062 & $0.990-1.140$ & 0.093 \\
\hline
\end{tabular}

HR, hazard ratio; CI, confidence interval; s-apelin, serum apelin; CTP score, Child-Turcotte-Pugh's score; MELD score, the Model for Endstage Liver Disease score; LSM, liver stiffness measurement; HVPG, hepatic venous pressure gradient.

${ }^{*}$ Cox regression hazard model; ${ }^{\dagger}$ Age- and sex-adjusted.
LSM (0.835), s-apelin (0.802), and HVPG (0.919), AUROC of s-apelin showed significantly better diagnostic ability than CTP score (CTP score vs s-apelin, $\mathrm{p}=0.006$ ). However, there was no difference in AUROC between s-apelin and MELD score and LSM (MELD score vs s-apelin, $\mathrm{p}=0.0878$ and LSM vs s-apelin, $\mathrm{p}=0.4231$ ). The AUROC of HVPG was better than that of s-apelin in prediction of cirrhosis (HVPG vs s-apelin, $\mathrm{p}=0.0061$ ) (Fig. $4 \mathrm{~A})$.

The mean HVPG value was $14.3 \pm 0.4 \mathrm{~mm} \mathrm{Hg}$. s-apelin also showed a significant linear correlation with HVPG $\left(R^{2}=0.356\right.$, $\mathrm{p}<0.001$ ) (Fig. 2B). In addition, in patients with CSPH, s-apelin was dramatically elevated compared with non-CSPH patients $(946.3 \pm 155.0 \mathrm{pg} / \mathrm{mL}$ vs $550.9 \pm 126.6 \mathrm{pg} / \mathrm{mL}, \mathrm{p}<0.001)$. The AUROCs for the prediction of CSPH of CTP score, MELD score, LSM and s-apelin were $0.775,0.793,0.924,0.962$, respectively. The AUROC of s-apelin showed significantly better diagnostic ability than those of CTP and MELD score $(\mathrm{p}<0.001)$ and was similar with the AUROC of LSM ( $p=0.108$ ) (Fig. 4B).

\section{4. s-apelin and the prediction of decompensation and mor- tality}

In the univariate analysis performed to select the factors that predict decompensation and mortality, s-apelin, CTP score, MELD score, LSM, and HVPG were all significantly associated with new first development of decompensation event and mortality $(\mathrm{p}<0.001)$. Among these factors, MELD was excluded in multivariate analysis because of multicollinearity with CTP score. In a multivariate analysis for the development of decompensation using a Cox regression hazard model about these 

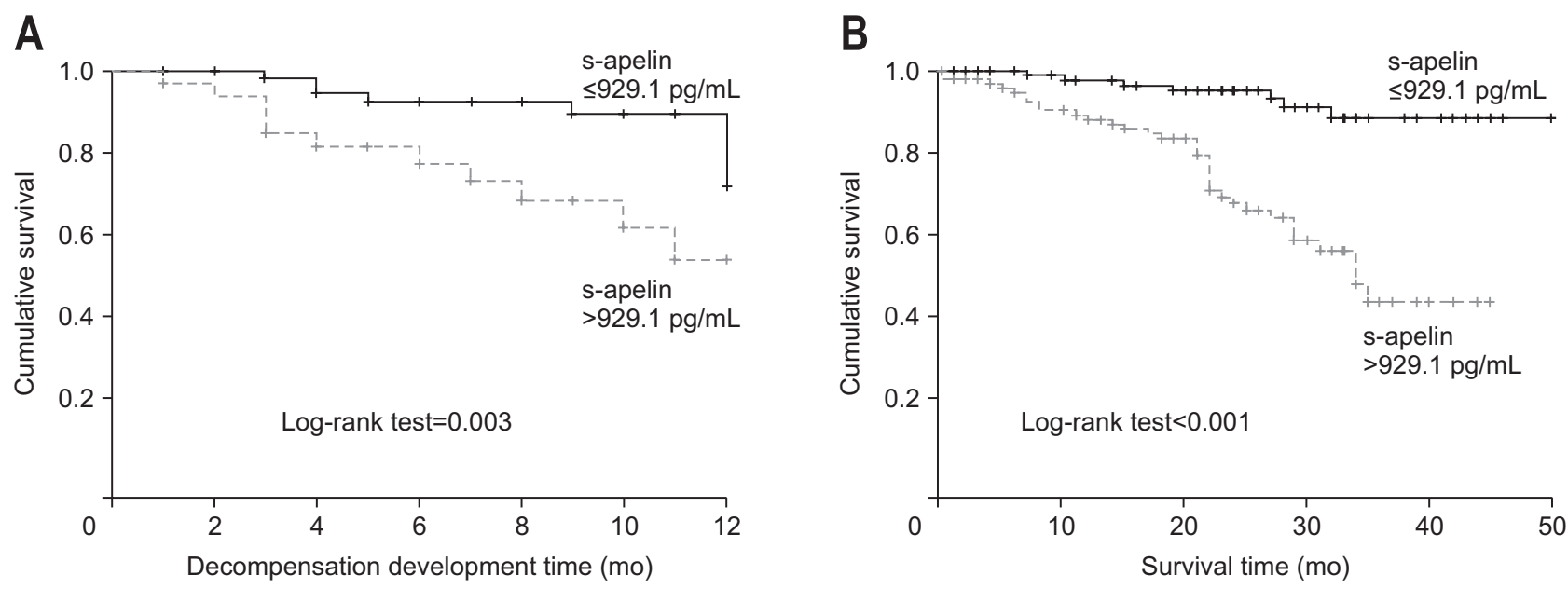

Fig. 5. The Kaplan-Meier analysis for the development of decompensation and mortality during the follow-up period. (A) Patients with a baseline serum apelin level (s-apelin) greater than $929.1 \mathrm{pg} / \mathrm{mL}$ had significantly shorter times to development of decompensation (log-rank test=0.003). (B) Patients with a baseline s-apelin greater than $929.1 \mathrm{pg} / \mathrm{mL}$ also had significantly shorter median survival times (34.0 \pm 2.4 months, log-rank test $<0.001)$.

factors, only s-apelin was a statistically significant variable predicting decompensation, with a hazard ratio of 1.002 (Table 2). In the Kaplan-Meier analysis performed to compare the subjects according to a median s-apelin of $929.1 \mathrm{pg} / \mathrm{mL}$, patients with an s-apelin greater than $929.1 \mathrm{pg} / \mathrm{mL}$ had a significant difference (p<0.001) (Fig. 5A).

In predicting mortality, s-apelin was also only a statistically significant variable predicting mortality in a Cox regression hazard model with a hazard ratio of 1.003 (Table 2). In the Kaplan-Meier analysis performed to compare the subjects according to a median s-apelin of $929.1 \mathrm{pg} / \mathrm{mL}$, patients with an s-apelin greater than $929.1 \mathrm{pg} / \mathrm{mL}$ had a significantly shorter mean survival time (34.0 \pm 2.4 months, $\mathrm{p}<0.001)$ (Fig. 5B).

Time dependent AUROC of s-apelin for the prediction of mortality in 1, 2, and 3 year were 0.641 (0.528 to 0.754), 0.708 (0.617 to 0.798 ), and 0.804 (0.68 to 0.927 ), respectively.

\section{DISCUSSION}

Through the previous preclinical studies, apelin has been known to have an important role in induction of hepatic fibrosis and vascular remodeling. In cirrhotic rat model experiment, apelin expression increased markedly in hepatic stellate cells, whereas apelin receptor was overexpressed in the hepatic parenchyma. Intrahepatic fibrosis and vessel density were diminished with the administration of apelin receptor antagonist. ${ }^{14}$ Apelin seems to behave as a hepatic paracrine substance, secreted by the activated hepatic stellate cell which was activated by some kind of stimulation such as hypoxia, endotoxin, or inflammatory factors. Through paracrine manner, apelin enhanced triggering the next steps of proinflammatory and neoangiogenic signaling pathways. ${ }^{14,16}$

Apelin also contributed to portosystemic collateralization and splanchnic neovascularization in portal hypertensive rats model, too. ${ }^{15}$ In this model, apelin and its receptor were overexpressed in the splanchnic vasculature. The administration of antagonist of apelin receptor (F13A) effectively decreased of splanchnic neovascularization and the formation of portosystemic collateral vessels as well as the expression of proangiogenic factors VEGF, PDGF and angiopoietin 2. These findings strongly suggested a possibility of apelin as a new therapeutic target in terms of both fibrosis and PHT. ${ }^{15}$

In spite of such a hopeful expectation, the validation data about the apelin in real clinical situation is very rare. Especially, the possibility as a noninvasive diagnostic biomarker for the severity of liver disease has not been studied. So, the present study is valuable that it evaluated whether the findings of apelin from preclinical study coincide with clinical practice and investigated the possibility of s-apelin as noninvasive biomarker. For these, this study analyzed the relationship and diagnostic values of apelin with both histological and hemodynamic severity respectively. And finally, we analyzed the prognostic value of s-apelin about the development of decompensation and mortality in the longitudinal cohort data.

First, in terms of histological assessment, s-apelin had a direct correlation with the degree of hepatic fibrosis in the present study. This result is the first data that shows the direct correlation between hepatic fibrosis histological grades and systemic apelin level in human. s-apelin showed significant linear correlation with not only indirectly measured fibrosis grade by LSM $\left(\mathrm{R}^{2}=0.263, \mathrm{p}<0.001\right)$ but also quantitative fibrosis amount which was directly measured by CPA $\left(\mathrm{R}^{2}=0.213, \mathrm{p}<0.001\right)$. s-apelin measurement also has an advantage in the point of noninvasiveness. It was safe and repeatable. However, the strength of correlation between s-apelin and histological grades was not so strong to make predictive model. Especially, diagnostic ability of 
s-apelin estimated by AUROC did not showed superiority with another noninvasive diagnostic method LSM which recently has been most strongly investigated as a noninvasive diagnostic method for hepatic fibrosis in the clinical field.

Second, this study showed the direct correlation between PHT measured by HVPG and s-apelin $\left(\mathrm{R}^{2}=0.356, \mathrm{p}<0.001\right)$. s-apelin showed increase according to the increase of portal pressure and this data is also novel. The diagnostic ability for CSPH was also better than traditional prognosis marker CTP and MELD score. Actually, in contrast LSM which just reflect intrahepatic change, s-apelin has relationship with both intrahepatic and splanchnic change in cirrhosis, so it was expected s-apelin could was able to have more powerful diagnostic value than LSM. However, sapelin's diagnostic value did not showed any additional benefit compared to LSM.

Finally, through the significant correlation with both histological and hemodynamic components, s-apelin showed a significant value as a prognostic biomarker for the development of decompensation and mortality. However, like histological and hemodynamic severity, the hazard ratios were very weak. In addition, time dependent AUROC of s-apelin for the prediction of mortality in relatively early period such as 1 and 2 year were not so satisfactory to use as prognostic model (0.641 [0.528 to 0.754], 0.708 [0.617 to 0.798]).

So, all these findings suggest that s-apelin absolutely has a significant relationship and role with the disease progression, but they also showed s-apelin alone has a limitation in clinical application. Many reasons can be considered for these findings. The opposite action of s-apelin can be one reason. As known well, apelin generally increase NO synthesis, however in the intrahepatic condition of cirrhosis, decrease of NO production is main pathogenesis of intrahepatic resistance. We do not know the exact action mechanism of apelin in the liver and some kind of signaling defect such as postreceptor dysfunction can make discrepancy between s-apelin level and the clinical manifestation. Especially, in the advanced cirrhosis, not only liver but also other organs including heart, kidney, lung show dysfunction and the expression of apelin and it can make a difficulty in interpretation of s-apelin data. Finally, as like so many other cytokines and biomarkers, s-apelin is just one of the numerous contributing factors of cirrhosis and it is impossible to explain the complexity of mechanism of cirrhosis and PHT with just apelin alone. In this line, the application of apelin receptor antagonist for histological or hemodynamic improvement which was suggested in the previous preclinical studies has limitation in clinical practice.

As far as authors know, this study is the first study that showed the clinical meaning and prognostic value of s-apelin in both histological and hemodynamic aspect of human CLD with longitudinal data. However, the present study has several limitations too. First, this analysis was designed retrospectively. Retrospective study is limited in developing novel diagnostic modalities or survival prediction models. The usefulness of sapelin as a prognostic biomarker should be confirmed by future prospective studies. Second, the included causes of liver cirrhosis were heterogeneous and the cohort could not show the etiology specific difference.

In conclusion, this study confirmed a clinically significant association between s-apelin and histological and hemodynamic state of CLD. However, s-apelin showed less strong possibility to be applied as a novel noninvasive prognostic biomarker in clinical practice. Hopefully, future well-designed prospective studies would be followed to validate the use of s-apelin in the clinical field.

\section{CONFLICTS OF INTEREST}

No potential conflict of interest relevant to this article was reported.

\section{ACKNOWLEDGEMENTS}

This work was supported by a research grant from the Yonsei University Wonju College of Medicine (YUWCM 2012-75175) and also by a grant of the Korea Health Technology R\&D Project, Ministry of Health and Welfare, Republic of Korea (A100054).

\section{REFERENCES}

1. Arvaniti V, D’Amico G, Fede G, et al. Infections in patients with cirrhosis increase mortality four-fold and should be used in determining prognosis. Gastroenterology 2010;139:1246-1256.

2. Garcia-Tsao G, Friedman S, Iredale J, Pinzani M. Now there are many (stages) where before there was one: in search of a pathophysiological classification of cirrhosis. Hepatology 2010;51:14451449.

3. Kim MY, Baik SK, Lee SS. Hemodynamic alterations in cirrhosis and portal hypertension. Korean J Hepatol 2010;16:347-352.

4. Park EJ, Jang JY, Lee JE, et al. The risk factors for bleeding of fundal varices in patients with liver cirrhosis. Gut Liver 2013;7:704711.

5. Lee TH, Han SH, Yang JD, Kim D, Ahmed M. Prediction of advanced fibrosis in nonalcoholic fatty liver disease: an enhanced model of BARD Score. Gut Liver 2013;7:323-328.

6. Garcia-Tsao G, Boyer JL. Outpatient liver biopsy: how safe is it? Ann Intern Med 1993;118:150-153.

7. Lebrec D. Methods to evaluate portal hypertension. Gastroenterol Clin North Am 1992;21:41-59.

8. Kim MY, Suk KT, Baik SK, et al. Hepatic vein arrival time as assessed by contrast-enhanced ultrasonography is useful for the assessment of portal hypertension in compensated cirrhosis. Hepatology 2012;56:1053-1062.

9. O’Dowd BF, Heiber M, Chan A, et al. A human gene that shows 
identity with the gene encoding the angiotensin receptor is located on chromosome 11. Gene 1993;136:355-360.

10. Tatemoto K, Hosoya M, Habata Y, et al. Isolation and characterization of a novel endogenous peptide ligand for the human APJ receptor. Biochem Biophys Res Commun 1998;251:471-476.

11. Ishida J, Hashimoto T, Hashimoto Y, et al. Regulatory roles for APJ, a seven-transmembrane receptor related to angiotensin-type 1 receptor in blood pressure in vivo. J Biol Chem 2004;279:2627426279.

12. Tatemoto K, Takayama K, Zou MX, et al. The novel peptide apelin lowers blood pressure via a nitric oxide-dependent mechanism. Regul Pept 2001;99:87-92.

13. De Mota N, Reaux-Le Goazigo A, et al. Apelin, a potent diuretic neuropeptide counteracting vasopressin actions through inhibition of vasopressin neuron activity and vasopressin release. Proc Natl Acad Sci U S A 2004;101:10464-10469.

14. Principe A, Melgar-Lesmes P, Fernández-Varo G, et al. The hepatic apelin system: a new therapeutic target for liver disease. Hepatology 2008;48:1193-1201.

15. Tiani C, Garcia-Pras E, Mejias M, et al. Apelin signaling modulates splanchnic angiogenesis and portosystemic collateral vessel formation in rats with portal hypertension. J Hepatol 2009;50:296-
305.

16. Melgar-Lesmes P, Pauta M, Reichenbach V, et al. Hypoxia and proinflammatory factors upregulate apelin receptor expression in human stellate cells and hepatocytes. Gut 2011;60:1404-1411.

17. Kim MY, Baik SK, Yea CJ, et al. Hepatic venous pressure gradient can predict the development of hepatocellular carcinoma and hyponatremia in decompensated alcoholic cirrhosis. Eur J Gastroenterol Hepatol 2009;21:1241-1246.

18. de Franchis R. Updating consensus in portal hypertension: report of the Baveno III Consensus Workshop on definitions, methodology and therapeutic strategies in portal hypertension. J Hepatol 2000;33:846-852.

19. Hong WK, Kim MY, Baik SK, et al. The usefulness of non-invasive liver stiffness measurements in predicting clinically significant portal hypertension in cirrhotic patients: Korean data. Clin Mol Hepatol 2013;19:370-375.

20. Kim MY, Jeong WK, Baik SK. Invasive and non-invasive diagnosis of cirrhosis and portal hypertension. World J Gastroenterol 2014;20:4300-4315.

21. Tsochatzis E, Bruno S, Isgro G, et al. Collagen proportionate area is superior to other histological methods for sub-classifying cirrhosis and determining prognosis. J Hepatol 2014;60:948-954. 